\title{
Utilizing the full Capacity of Sorting Systems with new Infeed Technology
}

\author{
Volker Sadowsky \\ Kai F. Semrau \\ Chair of Materials Handling and Warehousing (FLW) \\ TU Dortmund University, Germany
}

$\mathbf{U}$ tilisation of sorting systems' maximum performance demands continuous conveying of goods by infeed lines to sorters. This is the case especially for sorters with one single infeed line, because the sorters' performance is limited by the performance of the infeed line. Within this paper different infeed line constructions at the Rotary Sorter with diverse performances will be presented. The focus lies on a specific conveying system to synchronise the goods with the sorter by a performance of 6000 pieces per hour with one dynamic infeed line. By this means there is no extensive adjustment control of serial conveyors in the infeed line any longer.

[Keywords: Drehsorter, rotary sorter, infeed, sorter, sorting, SpinSorter]

\section{INTRODUCTION}

The infeed of items in sorting systems is a demanding technical control task and an essential factor for the optimization of sorting systems. This especially applies to such sorting systems where there is space for only one infeed line. In these cases the effective performance of the sorter is a direct result of the infeed capacity. For sorters with only one infeed line the infeed capacity has a wide impact on the overall performance of a sorting system and therefore on the performance of the whole logistics system.

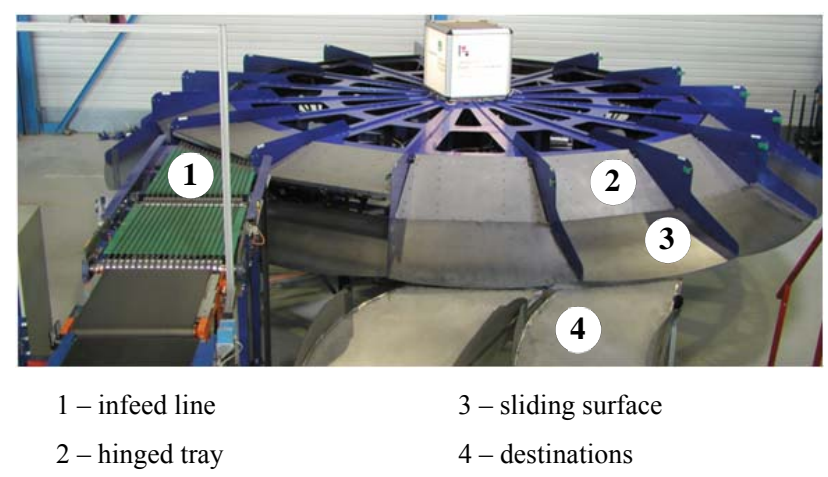

Figure 1. Rotary sorter prototype

Sorting systems are, for example, used to distribute and sort goods in distribution or logistics centers. They al- low the distribution of randomly arriving goods to several destinations by given distinctive features [VDI3619]. The central element of a sorting system is called sorter [JH06]. It consists of infeeds, which are responsible for transferring the goods from conveyor belts to the distribution conveyor, and of destinations to which the goods are sorted (fig. 1).

Sorters are used in different branches and therefore they are subject to various performance requirements. Moreover, the different goods have to be handled safely. For that reason, there are many differently designed sorters with a wide performance spectrum on the market. In the segment with the lowest performance level, up to 5000 items/hour, pushers and transfer systems are preferably used. Till-tray sorters, cross-belt sorters and sliding-shoe sorters, all known as high speed sorters, dominate the sector with a performance level above 5000 items/h [JH06].

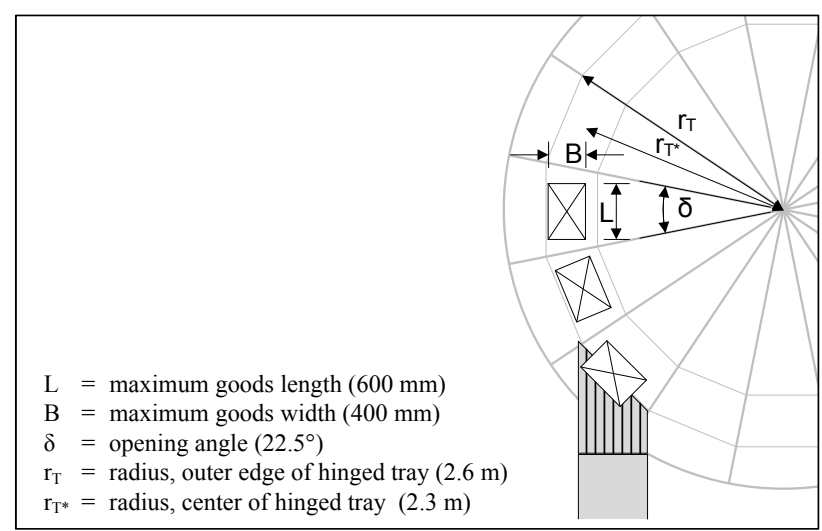

Figure 2. Geometrical relations of prototype (topview)

The rotary sorter (fig. 1) developed by the Chair of Materials Handling and Warehousing (FLW) at TU Dortmund University and the Fraunhofer Institute for Material Flow and Logistics (IML) was designed with the goal to lower investment and running costs for the medium performance level. The rotary sorter prototype at FLW is suitable for sorting and distributing items with a maximum length of $600 \mathrm{~mm}$ (fig. 2). In the prototype different infeeds and discharges were implemented to investigate the kinetic behavior of items during infeed into the sorter and during discharge via the rotating sliding surface. 


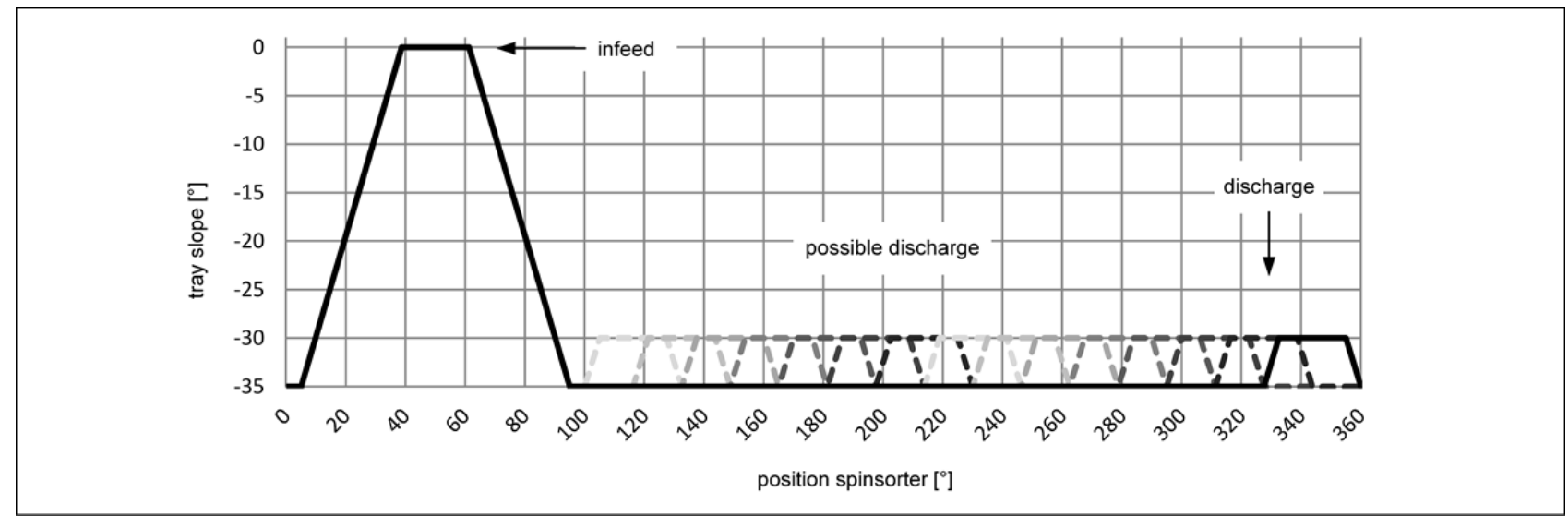

Figure 3. Tray slope during one rotary sorter cycle

The core element of the rotary sorter is the rotating, truncated plate which conveys items in its 16 pivoting trays to their external destinations (fig. 4a). Outside the hinged tray there is a rotating sliding surface which is part of the rotating plate. The goods slide across this surface during discharge before they reach the destinations. The infeed can be designed as a horizontal angle infeed (fig. 1, fig. 2) or as an overhead infeed (fig. 4a, 4b). In the infeed area a mechanical device leads the hinged trays into a horizontal position $\left(0^{\circ}\right)$ to pick up goods (fig. 3 ).

After feeding the items into the rotary sorter the same mechanical device lowers the hinged tray so far that its
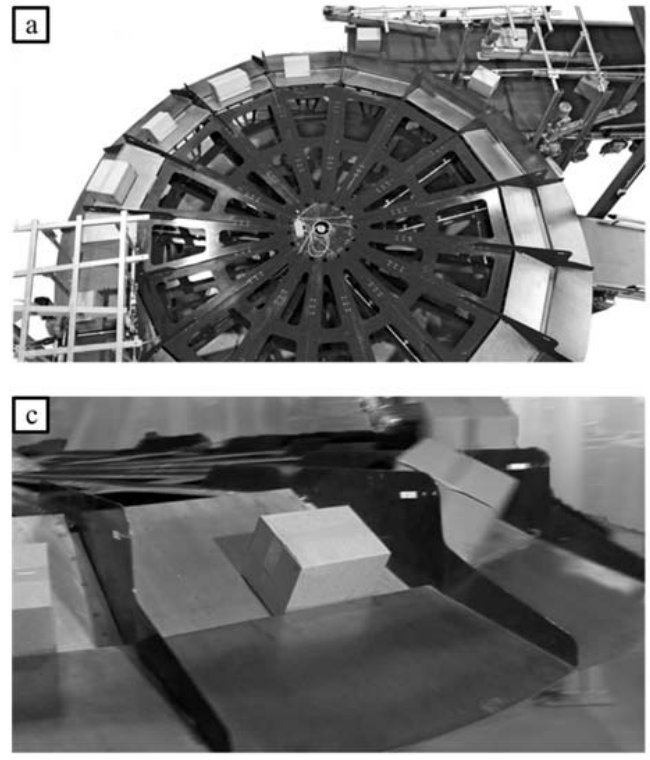

Figure 4. Sorting with rotary sorter ( $a$ - construction; $b$-infeed; $c$ - conveying; $d$-discharge)

With the above shown prototype and the described movement procedure it is possible to sort items with a maximum size of $600 \mathrm{~mm} \times 400 \mathrm{~mm} \times 400 \mathrm{~mm}$ (length/ width/height) and a maximum weight of $15 \mathrm{~kg}$. With its current structure the prototype reaches an angular velocity $\omega$ of $0.651 / \mathrm{s}$ or a rotation speed $n$ of 375 rotations per hour. With its 16 segments $n_{S}$ the prototype offers a theoretical performance $\mathrm{P}$ of 6000 items $/ \mathrm{h}$ : external edge is beneath the rotating sliding surface (slope $30^{\circ}$ ) (fig. 4c). By pneumatically raising the hinged trays by $5^{\circ}$ (micro throw) until they show the same slope as the sliding surface, the items are discharged (fig. 4d). Accelerated by gravity and centrifugal force (relation of about $7: 1$ with mass $\mathrm{m}=15 \mathrm{~kg}$, performance $\mathrm{P}=5000$ items $/ \mathrm{h}$ ), they slide over the rotating sliding surface to their destination. As the rotary sorter continues to rotate while the items are sliding, it is necessary to lift the hinged trays before the items reach the destination to include the offset of the rotation angle [Sch00].
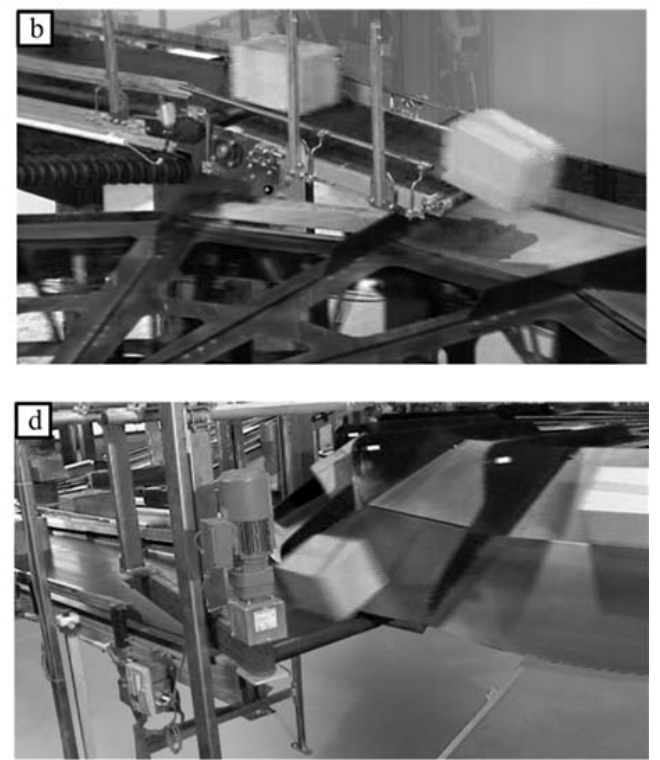

$$
P=\frac{\omega \cdot n_{S}}{2 \pi}=n \cdot n_{S} \rightarrow 375 \frac{1}{h} \cdot 16=6000 \frac{1}{h}
$$

With this performance the destinations - different chutes and conveyors - are accurately hit. To actually reach this sorting performance the infeed has to be structured in such a way that it can accurately feed 6000 items/h into the sorter. In this paper it is described how this infeed can be implemented. 


\section{LATEST State OF ART}

After patenting the rotary sorter in 1997 , Schmidt introduced the rotary sorter concept based on a theoretic sorting performance of $7000 \mathrm{items} / \mathrm{h}$ and items with a dimension of maximal $600 \mathrm{~mm}$ x $400 \mathrm{~mm}$ (length $\mathrm{x}$ width) [Sch97]. More details and special features like the effects caused by variation of goods length, angular velocity and segment number on the radius and the sorting performance were presented in 1999 [Sch99]. In his paper Schmidt points out that besides the mentioned parameters, the sorting performance also depends on the number and design of the infeeds. In the first extensive theoretic consideration of kinetic behavior and system performance Schmidt assumes a theoretic performance of 2400 to 7200 items/h [Sch00]. This consideration is based on the assumption that an infeed performance of 2400 items $/ \mathrm{h}$ is the maximum performance of a single stop-and-go infeed. By installing several infeed lines the infeed performance is multiplied, so that in case of three infeeds an overall infeed performance of 7200 items/h could be realized. The assumption of installing up to three infeeds is based on the strong reduction of destinations which each additional infeed line inevitably brings along. The number of possible destinations is limited by the circumference. As this number is low compared to sorters in loop or line structure, the realization and performance increase of one infeed line was focused.

In 2002 a prototype of the rotary sorter was accomplished and presented at the intralogistics trade fair CeMAT. The design of the prototype was realized with a horizontal angle infeed. With this infeed line the functionality of the rotary sorter concept could be verified. Since 2002 the prototype is located in the laboratory of FLW in Dortmund and Lödige Industries holds the licensing rights and markets the rotary sorter under the name SpinSorter.

The distribution performance of the rotary sorter with just one infeed was specified with 5000 items/h by ten Hompel and Schmidt in 2002. Even in the same year they emphasized the relevance of preparing material flows and synchronizing the goods with the sorter for system optimization [HS02b]. Their statements underlined the importance of the infeed in terms of utilizing the full capacity of sorting systems. Consequently, the kinetic behavior of goods during infeed was analyzed to determine performance criteria. In this context Hirschmüller stated in 2003 that with a higher infeed performance the rotary sorter could convey more than $6000 \mathrm{items} / \mathrm{h}$. He identified the development of a more efficient infeed technology and adequate control strategies as requirements for the utilization of the maximal performance of a rotary sorter [Hir03, SH03]. Subsequent examinations include the optimization of the kinetic behavior of the goods during the infeed to convey the items accurately onto the hinged trays even at high speeds [HM04, HJ04]. Here performances of up to 4000 items/h with one infeed were achieved during a short run. The performance was restricted by the used infeed technology. Thereby two variants - manual and automatic infeed - were examined. With one manual infeed a performance of 2400 items/h could be achieved. In contrast, one automatic infeed achieved according to the stop and go procedure the described performance of 4000 items/h [HM04].

\section{REDESIGN OF THE INFEED AREA}

The performance limiting features of the existing infeed technology led to the demand for an automatic infeed technology which can guarantee a continuous infeed to the rotary sorter with more than 4000 items $/ \mathrm{h}$. Therefore the prototype used at the Chair of Materials Handling and Warehousing, was extended by a circular closed continuous conveying system with new infeed technology in 2009 (fig. 5). The resulting circuit conveys the goods discharged by the rotary sorter (spinsorter) back to the redesigned infeed, from where they are directly inserted into the sorter again [SS10]. Therefore, the continuous conveying circuit offers the possibility to explore the performance of the rotary sorter in endurance testing.

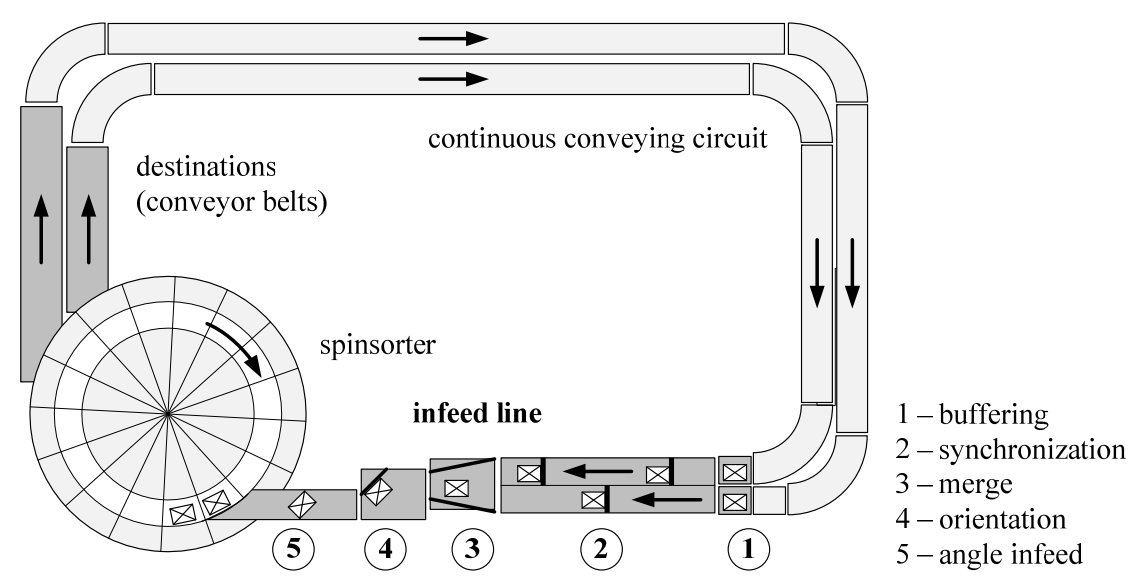

Figure 5. Rotary sorter with continuous conveying circuit and newly designed infeed line 
For the design of the infeed two essential requirements were formulated:

- An infeed performance of 6000 items/h has to be achieved for utilizing the full capacity of the rotary sorter.

- Arriving items have to reach the infeed area at an irregular distance, as it is in practice if the goods are put manually on the infeed.

To meet these requirements, a continuous conveying circuit including a new infeed with an improved performance was realized in cooperation with the companies Lödige Industries, Transnorm and AKDV (fig. 5).

Rotating the sorter clockwise conveys the goods to the destinations - two horizontally running belt conveyors - whereby the goods are conveyed within the continuous conveying circuit. By the horizontally running belt conveyors, arranged in two parallel lines, the goods are conveyed back to infeed area. The resulting continuous conveyor circuit enables the goods to be returned and the infeed performance to be examined in endurance tests.

The infeed begins with buffering the goods on two short conveyors (fig. 5, station 1). From there the items are fed to a synchronization conveyor (positioner) to be synchronized with the rotary sorter (fig. 5, station 2). After merging the two material flows (fig. 5, station 3) the goods are aligned in an angle of $45^{\circ}$ at a powered deflector belt positioned vertically above the conveyor (fig. 5, station 4). The items' $45^{\circ}$ orientation is required for the angle infeed belt (fig. 5, station 5). In the following sections the three essential processes for a goods protecting and precise infeed are explained in more detail.

\subsection{SYNCHRONIZATION}

To precisely feed the items to the hinged trays the rotary sorter has to be positioned in a precisely defined way at the time of the infeed. On the one hand, this is achieved by a strictly predefined relation of velocity of rotary sorter and the infeed. On the other hand, the goods have to arrive at the front edge of the induction belt at the exactly same moment as the sorter segment reaches a strictly predefined position in relation to the induction belt so that the goods can be dynamically transferred.

Therefore the synchronization conveyor (positioner) is used (fig. 6). The positioner consists of two parallel, not powered roller conveyors and three powered chains with square profiles (drivers) in between. The drivers are mounted to both conveying lines in identical intervals and circle the roller track. When goods are transferred from the buffering belts to the positioner, the goods freewheel at first. Subsequently they are caught from behind by a rotating driver and transported positively to the end of positioner. Thus the items are positioned in identical distance (back edge to back edge) which is strictly defined by the driver.

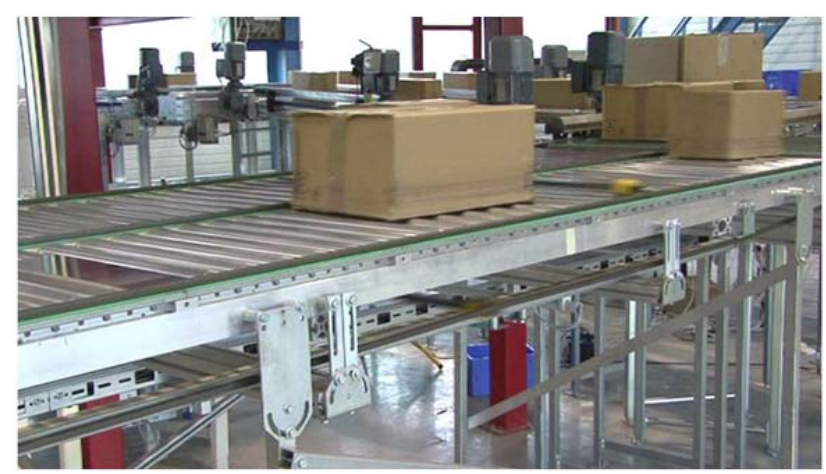

Figure 6. Synchronization conveyor (positioner), by Tansnorm System

The merging of the material flows will be done in zipper mode with a merge conveyor after positioning, as the drivers are mounted alternating on the two parallel lines before (fig. 5, station 2). The goods in the resulting material flow are conveyed with a constant velocity and a strictly predefined distance between the back edges of all packages. Due to the positive connection between drivers and goods, the synchronization of drivers and sorter segments' positions leads to the described effects.

To synchronize rotary sorter and driver, the positions of sorter segments and velocity of rotary sorter are measured at first. As soon as the data has reached rotary sorter control, a sync-impulse is transmitted to the drivers' control via TCP/IP. Subsequently the positioner or rather its chains with the drivers accelerate so that they match the rotary sorter performance. The positions of the sorter segments as well as the positions of the drivers are continuously determined by incremental encoders. The positioner accelerates as long as the positions of drivers and sorter segments are synchronized. During operation synchronization is ensured by a control circuit. Therefore the described procedure is only necessary once after starting the system or after setting a new velocity level.

\subsection{Alignment}

For an optimal use of space in the tray segments the packages have to be aligned before the infeed process. The alignment is done via a powered belt deflector which is vertically mounted above a belt conveyor in an approximately $45^{\circ}$ angle to conveyor direction (fig. 7). During the deflecting process the goods are moved with a constant belt velocity towards the deflector at first, until their front side hits the deflector. After the first collision the goods move with combined rotation and translation or just rotation. Due to the rotation the goods rotate until they lie flat against the deflector. Depending on deflector belts' velocity and direction of movement, the goods subsequently perform a translational movement. During these movement phases motion quantities of goods are changed [Mül80, Möh83]. 


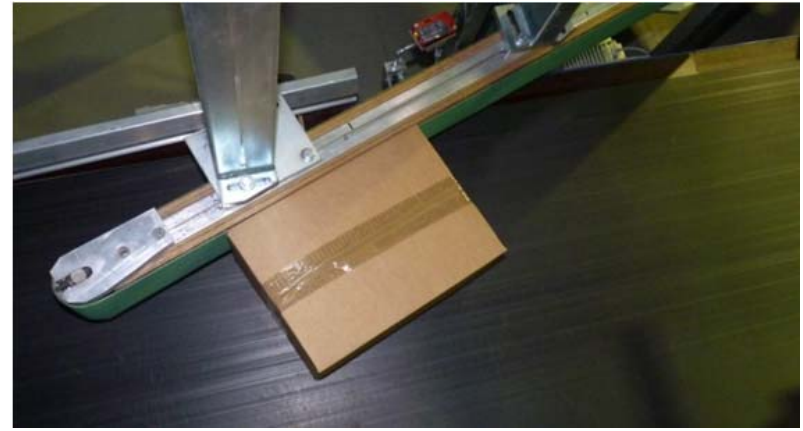

Figure 7. Orientation via belt deflector (topview)

As the described structure does not show significant differences between kinetic behaviors of different items at deflector belt, the synchronization between positioner and rotary sorter can be done regardless of the used items.

\subsection{ANGLE INFEED}

The horizontal transfer from infeed belt to rotary sorter guarantees a goods protecting infeed. For the transfer, an angle of $45^{\circ}$ between infeed belt and rotary sorter was chosen (fig. 8). In case of a bigger infeed angle the infeed velocity and the radial velocity are too high to match the tangential velocity. In contrast, a smaller infeed angle would reduce the radial velocity so that the goods are not fed to the hinged tray far enough. The transfer to the rotary sorter is realized by an induction conveyor with round belts.
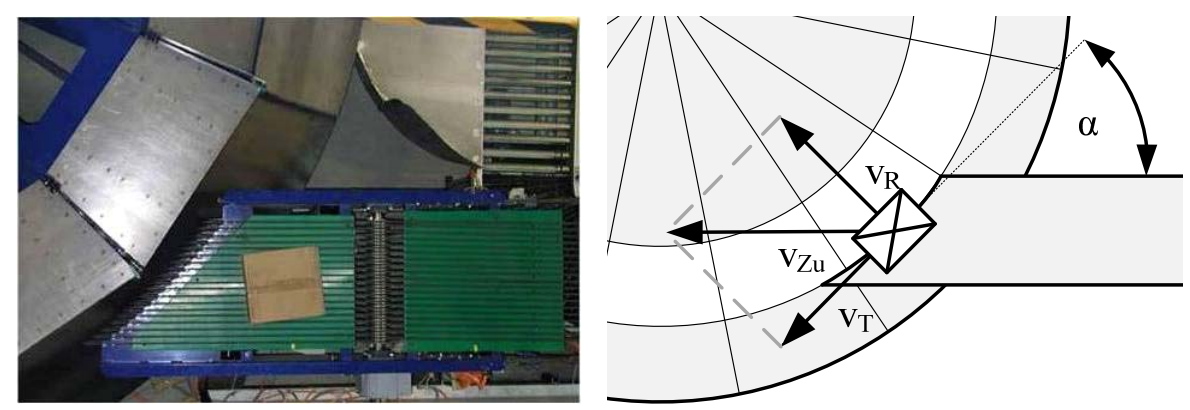

$\mathrm{v}_{\mathrm{Zu}}=$ infeed velocity
$\mathrm{v}_{\mathrm{T}}=$ tangential velocity
$\mathrm{v}_{\mathrm{R}}=$ radial velocity
$\alpha=$ infeed angle

Figure 8. Infeed angle and infeed velocity, according to [Hir03]

When the items are transferred to the sorter segments in a $45^{\circ}$ angle, they are positioned with their long side parallel to the side of the hinged tray. This is an important requirement for a precise discharge [Hir03].

In case of the horizontal angle infeed, it is important to consider that the velocity of the infeed belt has to be so high that the tangential velocity component of the items $\mathrm{v}_{\mathrm{T}}$ matches the tangential velocity of the rotary sorter. The correlation of velocities in case of an infeed with an angle $\alpha$ according to figure 6 is the result of:

$$
v_{Z u}=\frac{v_{T}}{\cos (\alpha)}
$$

The rotary sorter prototype rotates with an angular velocity $\omega$ of $0.651 / \mathrm{s}$ and has a radius $r_{T}$ of $2.6 \mathrm{~m}$ at the tray's outer edge. The result is a tangential velocity $\mathrm{v}_{\mathrm{T}}$ of $1.7 \mathrm{~m} / \mathrm{s}$ and a performance $\mathrm{P}$ of $6000 \mathrm{items} / \mathrm{h}$.

$$
v_{T}=\omega \cdot r_{T} \rightarrow 0.65 \frac{1}{\mathrm{~s}} \cdot 2.6 \mathrm{~m}=1.7 \frac{\mathrm{m}}{\mathrm{s}}
$$

This means that with a $45^{\circ}$ angle infeed the infeed velocity $\mathrm{v}_{\mathrm{Zu}}$ has to be at least

$$
v_{Z u}=\frac{v_{T}}{\cos (\alpha)} \rightarrow \frac{1.7 \frac{\mathrm{m}}{\mathrm{s}}}{\cos \left(45^{\circ}\right)}=2.4 \frac{\mathrm{m}}{\mathrm{s}}
$$

to feed the goods in a $45^{\circ}$ angle to the rotary sorter. But at this point it is necessary to consider that the velocity of items during transfer to the rotary sorter continuously changes. Tangential and radial velocities are continuously reduced by dynamic friction just as the velocity components of the rotary sorter because of the decreasing radius [Hir03].

Another important factor for determining the infeed velocity $\mathrm{V}_{\mathrm{Zu}}$ is the deviation in $90^{\circ}$ to infeed direction resulting from the different goods width. Based on the theoretic and experimental results of Hirschmüller [Hir03] and other experiments the value for the infeed velocity $\mathrm{v}^{\prime} \mathrm{Zu}$ was defined as

$$
v_{Z u}^{\prime}=2.6 \frac{\mathrm{m}}{\mathrm{s}}
$$

Thus the infeed velocity $\mathrm{v}^{\prime} \mathrm{Zu}$ and performance $\mathrm{P}$ are given for the infeed area, so that the constant goods distance $\mathrm{s}_{0}$ in the infeed area can be calculated.

$$
s_{0}=\frac{v_{Z u}}{P} \rightarrow \frac{2.6 \frac{\mathrm{m}}{\mathrm{s}}}{6000 \frac{1}{h}}=1.6 \mathrm{~m}
$$

The distance of $1.6 \mathrm{~m}$ corresponds with the distance between two drivers mounted alternated on the positioner and which define the distance between two items (fig. 9). 


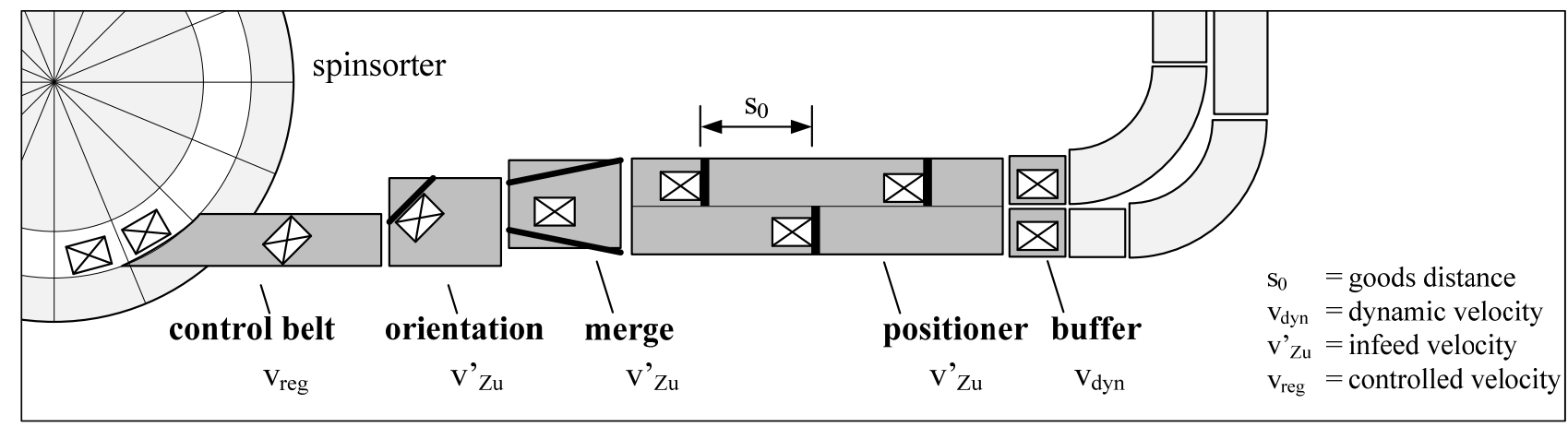

Figure 9. Schematic infeed line with angle infeed

\subsection{Test Results REgarding ANGLE INFEed}

By combining buffer belts, positioners, merge, alignment and control belt an innovative infeed technology has been designed and realized which enables the flawless feed of block-shaped items with a maximum length of $600 \mathrm{~mm}$ to the rotary sorter. For that purpose all conveyors in the infeed area were equipped with adequate electrical drives to increase the velocity up to $2.6 \mathrm{~m} / \mathrm{s}$ and to enable the performance of 6000 items $/ \mathrm{h}$.

In the performance area up to 4000 items/h the goods are exactly transferred to the tray segments of the rotary sorter. For this purpose the positioner positively synchronizes the goods with the rotary sorter. Synchronization is not substantially effected by merging or aligning process. The goods are continuously transported to the positioner, so that all gaps between the drivers of the positioner and therefore all sorter segments are filled. Through this process the infeed performance and the rotary sorter performance of 4000 items/h are fully utilized.

With the adjusted performances of 5000 and 6000 items/h the goods are still transferred from the buffer belts to the positioner and by positioner synchronously conveyed to the rotary sorter. However, at the deflector belt the goods are not sufficiently aligned in this high performance area. On the one hand the misalignment is a result of the high traction force in conveying direction which is caused by the high velocity of the conveyor belt so that the goods are pulled again into the longitudinal direction after hitting the deflector. On the other hand the goods hit the deflector with high velocity causing a translational deviation in $90^{\circ}$ to the conveying direction. These two effects impede a safe and precise infeed for the intended spectrum of block-shaped items.

To realize the performance of 6000 pieced per hour in the infeed area, there are two possibilities for the change of infeed. On the one hand it can be tried to improve the alignment technology to accomplish an orientation of goods in a $45^{\circ}$ angle with a conveyor belt velocity of $2.6 \mathrm{~m} / \mathrm{s}$. On the other hand there is the possibility to feed the goods into the rotary sorter tangentially from the top (overhead infeed).

\section{VARIATIONS OF INFEED TECHNOLOGY}

The infeed technology presented in chapter 3 does not meet the requirements due to insufficient alignment of the items at high velocities. Therefore, an overhead infeed was realized, where the goods do not have to be aligned in a $45^{\circ}$ angle to reach a conveyor belt velocity of $2.6 \mathrm{~m} / \mathrm{s}$, as they are transferred longitudinally aligned and tangentially from the top onto the rotary sorter (fig. 10).
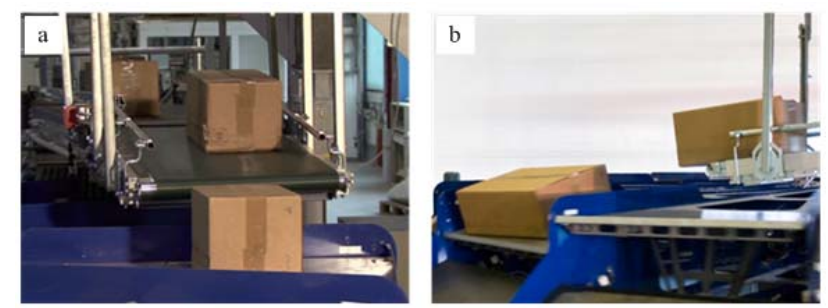

Figure 10. Overhead infeed, a) front view, b) side view

\subsection{OVERHEAD INFEED}

The overhead infeed enables the goods to be transferred in tangential movement direction of the rotary sorter, making the $45^{\circ}$ alignment redundant so that the packages can be fed in longitudinal (fig. 11). Due to the overhead infeed, the mechanical structure of the conveyor technology is changed so that the conveyors in the infeed area are tangentially arranged to the rotary sorter and that a height adjustment of these conveyors becomes necessary because of the infeed from the top. Furthermore the fragmentation of the velocity components, as described in chapter 3.3, is not necessary anymore. In case of overhead infeed the infeed velocity of the items $\mathrm{V}_{\mathrm{Zu}^{*}}$ corresponds exactly with the tangential velocity of the rotary sorter in the center of the tray segment $\mathrm{v}_{\mathrm{T}^{*}}$ (fig. 11). Therefore the tangential velocity $\mathrm{v}_{\mathrm{T}^{*}}$ can be distinguished from the tangential velocity of the rotary sorter at the edge of the tray segment $\mathrm{v}_{\mathrm{T}}$, as described in chapter 3.3. 


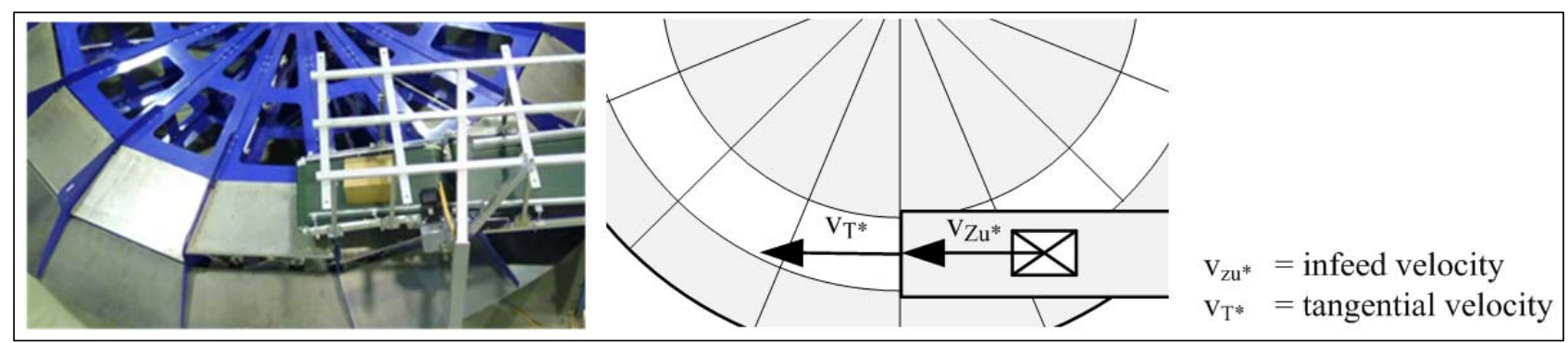

Figure 11. Overhead infeed and infeed velocity

For equation 3.1 in case of overhead infeed with an infeed angle $\alpha=0$ the following is to be applied:

$$
v_{Z u^{*}}=\frac{v_{T^{*}}}{\cos (\alpha)} \rightarrow \frac{v_{T^{*}}}{\cos (0)}=v_{T^{*}}
$$

For the performance of 6000 items/h the tangential velocity $\mathrm{v}_{\mathrm{T}^{*}}$ of the tray center of the rotary sorter is $1.5 \mathrm{~m} / \mathrm{s}$ with a radius $r_{\mathrm{T}^{*}}$ of $2.3 \mathrm{~m}$.

$$
v_{T^{*}}=\omega \cdot r_{T^{*}} \rightarrow 0.65 \frac{1}{\mathrm{~s}} \cdot 2.3 \mathrm{~m}=1.5 \frac{\mathrm{m}}{\mathrm{s}}
$$

The tangential velocity $\mathrm{v}_{\mathrm{T}} *$ corresponds with the infeed velocity $\mathrm{V}_{\mathrm{Zu}^{*}}$ in case of the overhead infeed, according to the equation 4.1 has to be applied:

$$
v_{Z u^{*}}=1.5 \frac{\mathrm{m}}{\mathrm{s}}
$$

As the velocity of rotary sorter (tray center) and the velocity of items on the last infeed belt have to be identical, so that goods can exactly fed in the hinged trays of rotary sorter, the distance between goods on both conveyors has to be the same. This distance is exactly the length of a circular arc element $s_{1}$ at the radius $r_{T^{*}}$. With the infeed velocity $\mathrm{v}_{\mathrm{Zu}^{*}}$ and the distance $\mathrm{s}_{1}$ the performance $\mathrm{P}$ can be determined.

$$
P=\frac{v_{Z u^{*}}}{s_{1}} \rightarrow \frac{1.5 \frac{m}{s}}{0.9 m}=6000 \frac{1}{h}
$$

Therefore, the distance between the goods is exactly adjusted to the distance of sorter segments on the circular arc of rotary sorter with a radius $\mathrm{r}_{\mathrm{T}^{*}}$ of $2.3 \mathrm{~m}$. This means, that the last infeed belt has to convey the goods with exactly the same velocity and the same distance as the rotary sorter. For that purpose the induction belt has to be slower than the previous ones, so that the distance between goods in front of the rotary sorter is reduced from $1.6 \mathrm{~m} \mathrm{(s0)}$ on the positioner to $0.9 \mathrm{~m} \mathrm{(s1)} \mathrm{during} \mathrm{infeed}$ (fig. 12).

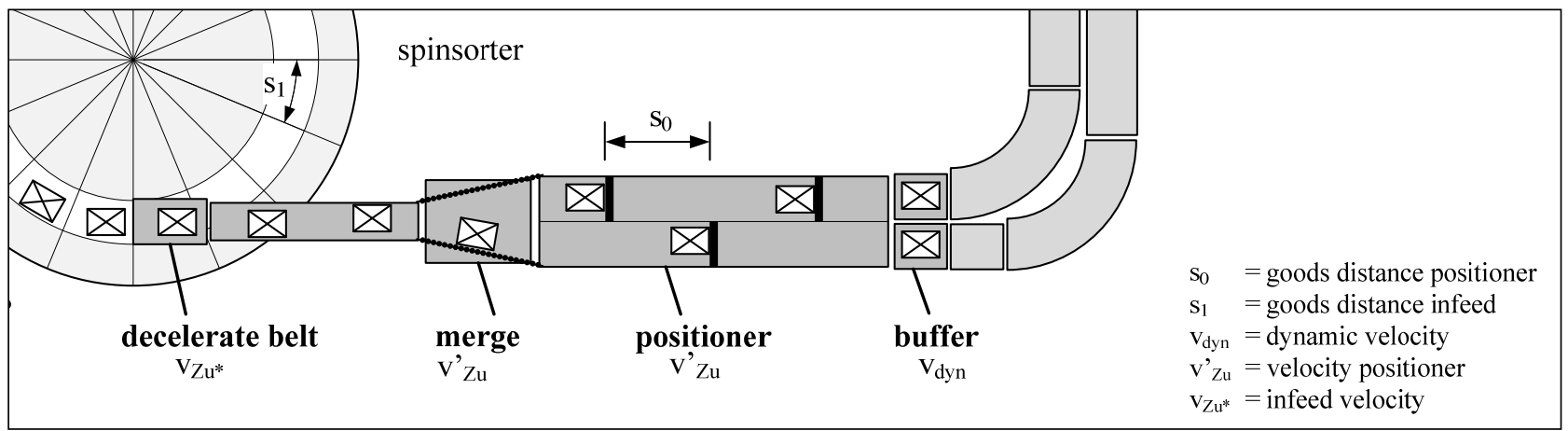

Figure 12. Schematic infeed line with overhead infeed

\subsection{TEST RESUlts REgARDING OVERHEAD INFEED}

The change of the infeed technology from angle infeed to overhead infeed brings along a change in the performance parameters. The distance of $1.6 \mathrm{~m}$ and a velocity of $2.6 \mathrm{~m} / \mathrm{s}$ created by the positioner are reduced to $0.9 \mathrm{~m}$ and $1.5 \mathrm{~m} / \mathrm{s}$ at the overhead infeed. The alignment of goods at a $45^{\circ}$ angle is not necessary anymore as the goods are fed aligned longitudinally.

The configuration with overhead infeed shows that the items are exactly placed at the center of the hinged trays despite the high deceleration on the induction belt.
The exact infeed is also be applied to the high performance levels, enabling performances up to $6000 \mathrm{items} / \mathrm{h}$.

In a one hour longing experiment a sorting performance of 5681 items/h could be reached with overhead infeed. In this experiment parcels made of double faced corrugated cardboard with a size of $310 \mathrm{~mm}$ x $220 \mathrm{~mm}$ x $200 \mathrm{~mm}$ and with a weight of approximately $3 \mathrm{~kg}$ were used. In short time view about 15 Parcels one after another were put onto the hinged trays of the rotary sorter without a gap. The gaps in the material flow are caused by the test facility as a conveying circuit whereby too little parcels are conveyed continuously to rotary sorter for a cer- 
tain time. Though control system problems happened, that caused material flow discontinuations.

\section{Conclusion ANd Prospect}

The goal of redesigning the infeed area of the rotary sorter prototype at the Chair of Materials Handling and Warehousing was the realization of an innovative infeed technology which enables 6000 items per hour to be fed in and therefore to utilize the full capacity of the rotary sorter with one single infeed. Overhead infeed and positioner are essential components of the new infeed area, enabling the goods to be synchronously conveyed and exactly placed in the hinged trays of the rotary sorter at maximum performance with ordinary technology.

Besides the overhead infeed the angle infeed, which requires a prior alignment of the items, is still in use. With the angle infeed a sorting performance of 4000 items $/ \mathrm{h}$ can be accomplished. At higher performance levels the technology for the alignment of the goods limits the performance. And that is the reason why angle infeed is not suitable for performances over $4000 \mathrm{items} / \mathrm{h}$.

When comparing the overhead infeed with the angle infeed, application fields and features have to be considered on a case-by-case basis for both infeed variants. The goods protecting transfer and recirculation possibility by the angle infeed is especially to be confronted with the overhead infeed without alignment and with higher performance.

With new infeed technology the evidence was adduced for the rotary sorter capacity of 6000 items/h with just one infeed line. Expanding the performance level opens up new future application fields for the rotary sorter. Currently other performance enhancing effects on sorting systems are examined by the Chair of Materials Handling and Warehousing.

\section{SYMBOLS}

Symbols SI units Name

\begin{tabular}{|c|c|c|}
\hline B & {$[\mathrm{m}]$} & goods width \\
\hline $\mathrm{L}$ & {$[\mathrm{m}]$} & goods length \\
\hline $\mathrm{P}$ & {$[1 / \mathrm{h}]$} & sorting performance \\
\hline $\mathrm{n}$ & {$[1 / \mathrm{h}]$} & rotation speed (rotations per hour) \\
\hline $\mathrm{n}_{\mathrm{S}}$ & {$[-]$} & $\begin{array}{l}\text { number of places (segments) on } \\
\text { the sorter }\end{array}$ \\
\hline $\mathrm{r}_{\mathrm{T}}$ & {$[\mathrm{m}]$} & radius (outer edge of hinged tray) \\
\hline $\mathrm{r}_{\mathrm{T}^{*}}$ & {$[\mathrm{~m}]$} & Radius (center of hinged tray) \\
\hline $\mathrm{s}_{0}$ & {$[\mathrm{~m}]$} & goods distance (positioner) \\
\hline $\mathrm{s}_{1}$ & [m] & goods distance (overhead infeed) \\
\hline $\mathrm{v}_{\mathrm{dyn}}$ & {$[\mathrm{m} / \mathrm{s}]$} & dynamic velocity \\
\hline $\mathrm{V}_{\mathrm{R}}$ & {$[\mathrm{m} / \mathrm{s}]$} & radial velocity \\
\hline $\mathrm{V}_{\text {reg }}$ & {$[\mathrm{m} / \mathrm{s}]$} & controlled velocity \\
\hline $\mathrm{v}_{\mathrm{T}}$ & {$[\mathrm{m} / \mathrm{s}]$} & $\begin{array}{l}\text { tangetial velocity (outer edge of } \\
\text { hinged tray) }\end{array}$ \\
\hline $\mathrm{V}_{\mathrm{T}^{*}}$ & {$[\mathrm{~m} / \mathrm{s}]$} & $\begin{array}{l}\text { tangetial velocity (center of } \\
\text { hinged tray) }\end{array}$ \\
\hline $\mathrm{V}_{\mathrm{Zu}}$ & {$[\mathrm{m} / \mathrm{s}]$} & $\begin{array}{l}\text { infeed velocity (angle infeed, the- } \\
\text { oretic) }\end{array}$ \\
\hline $\mathrm{V}_{\mathrm{Zu}^{*}}$ & {$[\mathrm{~m} / \mathrm{s}]$} & infeed velocity (overhead infeed) \\
\hline $\mathrm{v}^{6} \mathrm{Zu}$ & {$[\mathrm{m} / \mathrm{s}]$} & $\begin{array}{l}\text { infeed velocity (angle infeed, ex- } \\
\text { perimental) }\end{array}$ \\
\hline$\alpha$ & {$\left[{ }^{\circ}\right]$} & infeed angle \\
\hline$\delta$ & {$\left[{ }^{\circ}\right]$} & opening angle \\
\hline$\omega$ & {$[1 / \mathrm{s}]$} & angular velocity \\
\hline
\end{tabular}




\section{LITERATURE}

[Hir03] Hirschmüller, Thomas: Leistungskriterien und Systemfindung für die Stückguteinschleusung auf Sorter. Dissertation, Technische Universität Dortmund, 2003.

[HJ04]

[HM04]

[HS02a]

[HS02b]

[JH06]

[Möh83]

[Mül80]

ten Hompel, Michael; Jodin, Dirk: Leistungsreserven stecken im Detail - Kinematik der Sortiersysteme 2. Berlin: Huss-Medien GmbH. In: Hebezeuge und Fördermittel, Jahrgang 44, 11/ 2004, S.608-611.

ten Hompel, Michael; Mayer, Andreas: Strategy optimization at sorting system feeding station. In: Graduate School of Production Engineering and Logistics, Research Report. Technische Universität Dortmund. 2004, S.18-21.

ten Hompel, Michael; Schmidt, Thorsten: Das Drehsorterkonzept - Alternative zum Verteilen von Kleingut. Berlin: Huss-Medien GmbH. In: Hebezeuge und Fördermittel, Jahrgang 42, 2002, S.150-152.

ten Hompel, Michael; Schmidt, Thorsten: Sortier- und Verteilsysteme in Wissenschaft und Forschung. In: Fördern und Heben, Jahrgang 52, 9/ 2002, S.571-573.

Jodin, Dirk; ten Hompel, Michael: Sortier- und Verteilsysteme. Berlin Heidelberg: Springer-Verlag 2006.

Möhle, Bernd: Zum Bewegungsverhalten von Stückgütern auf Gurtförderern beim Ausrichten und Abweisen. Dissertation, Technische Universität Carolo-Wilhelmina zu Braunschweig, 1983.

Müller, Heinz: Untersuchungen zum Bewegungsverhalten von Einzellasten auf Gurtförderern beim Abweisvorgang. Dissertation, Technische Universität CaroloWilhelmina zu Braunschweig, 1980.

[Sch97] Schmidt, Thorsten: Rotieren und Sortieren. Fördertechnik '97, Sonderpublikation des Fördermittel Journals, Frankfurt/Main, 1997, S.36-37.

[Sch99] Schmidt, Thorsten: Rotierend verteilen. Berlin: Huss-Medien GmbH. In: Hebezeuge und Fördermittel, Jahrgang 39, 3/ 1999, S.74-75.

[Sch00] Schmidt, Thorsten: Stückgutverteilung nach dem Drehsorterkonzept - Bewegungsverhalten und Systemleistung. Dissertation, Technische Universität Dortmund, 2000.

[SH03] Schmidt, Thorsten; Hirschmüller, Thomas: Konzeption kostengünstiger Verteiltechniken am Beispiel des Drehsorters. In: Jahrbuch der Logistik, 2003, S. 237-241.

[SS10] Semrau, Kai F.; Sadowsky, Volker: Drehsorter neu aufgelegt - bis zu 6.000 Pakete/h eingeschleust und sortiert. In: Tagungsband auf CD zur 1. Central European Conference on Logistics, Miskolc, Ungarn; 2010.

[SW03a] Schmidt, Thorsten; Wölker, Christoph: Mit richtiger Technik und Software zum Erfolg. In: Logistik heute, Jahrgang 25, 4/ 2003, S.26-27.

[SW03b] Schmidt, Thorsten; Wölker, Christoph: Intelligent Sortieren. In: Hebezeuge und Fördermittel, Jahrgang 43, 5/ 2003, S.214-215.

[VDI3619] VDI 3619: Sortiersysteme für Stückgut. Berlin: Beuth-Verlag 1983. 